\title{
On the Friction Drag Reduction Effect by a Control of Large-Scale Turbulent Structures*
}

\author{
Koji FUKAGATA**, Michio KOBAYASHI*** and Nobuhide KASAGI*** \\ ${ }^{*}$ Department of Mechanical Engineering, Keio University \\ Hiyoshi 3-14-1, Kohoku-ku, Yokohama 223-8522, Japan \\ E-mail:fukagata@mech.keio.ac.jp \\ *** Depatment of Mechanical Engineering, The University of Tokyo \\ Hongo 7-3-1, Bunkyo-ku, Tokyo 113-8656, Japan
}

\begin{abstract}
Direct numerical simulation (DNS) of a feedback-controlled turbulent channel flow at $\mathrm{Re}_{\tau}=640$ is carried out. As an idealized feedback control, we selectively damp either the small scale wall-normal velocity fluctuations (defined as those with the spanwise wavelength smaller than 300 wall units) or the large scale fluctuations (the spanwise wavelength larger than 300 wall units). The present DNS reveals that the control of small scale fluctuations leads to more drag reduction than that of large scale fluctuations. When the small scale fluctuation is damped, the friction drag is reduced by the amount corresponding to the absence of small scale fluctuation. In contrast, when the large scale fluctuation is damped, the friction drag reduction is much less than that expected from the absence of large scale fluctuation. In the latter case, the contribution from the small scale fluctuation to the friction drag is found to be drastically increased due to the reduction of pressure fluctuation and destruction of Reynolds shear stress.
\end{abstract}

Key words: Turbulent Flow, Large-Scale Structure, Flow Control, Drag Reduction, Numerical Simulation

\section{Introduction}

Control of turbulence phenomena is one of the promising techniques for mitigating environmental impact and saving energy. Since the skin friction drag of wall turbulence causes large energy loss in many industrial devices, various control methods have been proposed to reduce it. For instance, Choi et al. ${ }^{(1)}$ demonstrated in their direct numerical simulation (DNS) of turbulent channel flow that $25 \%$ drag reduction could be achieved by local blowing/suction so as to oppose the wall-normal velocity just above the wall (v-control). DNS of similar control, which only uses the sensor information available on the wall, has also been reported. ${ }^{(2,3)}$

As an attempt to experimentally prove such feedback control of wall-turbulence, Yoshino et al. ${ }^{(4)}$ developed a feedback control system composed of arrayed micro wall-shear sensors and miniature magnetic actuators, and verified the drag reduction effect in their wind-tunnel experiment. Such systems require very small actuators (for instance, on the order of $100 \mu \mathrm{m}$ for airfoil application ${ }^{(5)}$ ), since the control is targeted at the viscous scale structure in the region near the wall. Fabrication and operation of such small-size devices, however, are formidable tasks even with the state-of-the art technology. Therefore, if possible, larger actuators are preferable for practical applications.

It is known that there are large-scale structures in high Reynolds number turbulence, which is the control target in the practical applications. For instance, Tomkins \& Adrian ${ }^{(6)}$ 
made PIV measurement of turbulent boundary layer at $R e_{\tau}=426$ and 2216. Based on the spanwise spectrum of streamwise velocity fluctuations, they found a large-scale structure of which spanwise wavelength of $0.8 \delta$. Abe et al. ${ }^{(7)}$ reported a large-scale mode of which spanwise wavelength of $1.3 \delta-1.6 \delta$ based on their DNS of channel flow up to $\operatorname{Re}_{\tau}=640$. Iwamoto et al. ${ }^{(8)}$ also reported a large-scale mode of which spanwise wavelength of $1.2 \delta$ in their DNS of channel flow at $R e_{\tau}=1160$ and 2320 .

In the previous studies on friction drag reduction in wall turbulence, effort has been made on suppression of the near-wall coherent structures. ${ }^{(1-5)}$ In high Reynolds number turbulence, however, the above-mentioned large-scale structures largely contribute to the friction drag. ${ }^{(8)}$ Although it has been theoretically predicted ${ }^{(9)}$ that in channel flows the drag reduction effect at high Reynolds number is roughly comparable to that at low Reynolds number even if only the near-wall coherent structures are suppressed, it is worthwhile to consider a different control target (i.e., large-scale structure), which is characteristic to the high Reynolds number flows.

In the present study, we investigate by means of numerical experiments using DNS the friction drag reduction effect by the suppression of large-scale structures, and obtain a basic turbulence control strategy toward the practical applications.

\section{Direct Numerical Simulation}

We consider a fully developed turbulent channel flow. The Reynolds number based on the bulk-mean velocity, $U_{\mathrm{b}}$, and the channel width, $2 \delta$, is $R e_{\mathrm{b}}=U_{\mathrm{b}}(2 \delta) / v=2.4 \times 10^{4}$. This corresponds to the friction Reynolds number of $R e_{\tau}=u_{\tau} \delta / v=2.4 \times 10^{4}=640$ in the uncontrolled flow (where $u_{\tau}$ denotes the friction velocity). The pseudo-spectral method similar to that of Kim et al. ${ }^{(10)}$ is used. Time integration is done by using the fourth order Runge-Kutta scheme for the advection term and the Crank-Nicolson scheme for the diffusion term. Spatial discretization is done by using the Fourier transformation in the streamwise $(x)$ and spanwise $(z)$ directions and the Chebyshev-tau method in the wall-normal $(y)$ direction. The flow rate is kept constant. The periodic boundary condition is adopted in the $x$ and $z$ directions, while no-slip condition is imposed on the walls.

The lengths of computational domain are $2.5 \pi \delta, 2 \delta$, and $\pi \delta$ in $x, y$ and $z$ directions, respectively. The numbers of spectral nodes are 288, 257 and 384, in $x, y$ and $z$ directions, respectively. The grid spacings are $\Delta x^{+}=17.7$ and $\Delta z^{+}=5.3$ in $x$ and $z$ directions, respectively. The minimum grid spacing in $y$ direction is $\Delta y_{\text {min }}^{+}=0.049$. Here, the superscript of + denotes the dimensionless value based on the friction velocity, $u_{\tau}$, of uncontrolled flow and the kinematic viscosity, $v$.

\section{Control Method}

In order to evaluate the drag reduction effect by an idealized suppression of large-scale structures, we apply a feedback body force, $-v / \tau$, so as to damp the specified wavenumber components of the wall-normal velocity $(v)$ in entire channel. Namely, the wall-normal momentum equation of the specified wavenumbers reads

$$
\frac{\partial v}{\partial t}=-(\vec{u} \cdot \nabla) v-\frac{1}{\rho} \frac{\partial p}{\partial y}+v \nabla^{2} v-\frac{v}{\tau}
$$

For ideal damping, the time constant, $\tau$, should be sufficiently small (in other words, the results may not significantly depend on its exact value if it is sufficiently small). Here, we set $\tau=10 v / u_{\tau}^{2}$. As will be shown later, this value gives a sufficiently strong damping. 


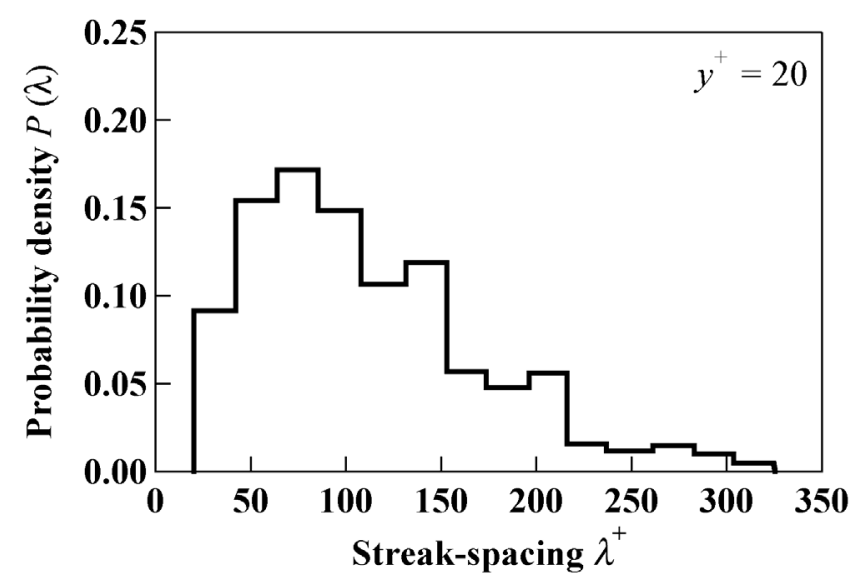

Fig. 1 Probability density histograms of spanwise streak spacing for $\mathrm{Re}_{\theta}=2030$ (Redrawn based on Smith \& Metzler ${ }^{(11)}$ )

As shown in Fig. 1, the spanwise spacing between the streaks, which is the representative near-wall structure, is experimentally shown to be less than $300 v / u_{\tau}{ }^{(8)} \mathrm{In}$ contrast, the spanwise size of the large-scale structure is on the order of $\delta$, as introduced above. Although the threshold wavelength between the small-scale and large-scale structures should ideally be set to a value much larger than the streak spacing and much smaller than $\delta$, these two scales are close to each other at the present Reynolds number, $R e_{\tau}$ $=640$. Therefore, in the present study, we conveniently classify the near-wall small-scale structures and the large-scale structures at the spanwise length of $300 v / u_{\tau}$ (i.e., about $0.5 \delta$ at $R e_{\tau}=640$ ). Hereafter, the fluctuation components of which spanwise wavelength is larger than $300 v / u_{\tau}$ is referred to as the large-scale fluctuations, while that less than $300 v / u_{\tau}$ is called the small-scale fluctuations. Note that no threshold is made for the streamwise wavelength, since both structures are relatively long in the streamwise direction. In the uncontrolled turbulent channel flow at $R e_{\tau}=640$, the small-scale and large-scale fluctuations share $1 / 3$ and $2 / 3$ of the total turbulent energy, respectively.

\section{Computational Results}

Figure 2 shows the time trace of the drag reduction rate, $R_{D}$, defined as

$$
R_{D}=1-\frac{(-\overline{d p / d x})_{\text {control }}}{(-\overline{d p / d x})_{\text {no control }}} .
$$

The drag reduction rates in the cases where the large-scale and small-scale fluctuations are damped (referred to as the large-scale damping and the small-scale damping) are $27 \%$ and $43 \%$, respectively, at their steady states.

The skin friction coefficient in a turbulent channel flow, $C_{f}$, is expressed by the summation of the laminar drag and the weighted integration of the Reynolds shear stress (RSS) $\left(-\overline{u^{\prime} v^{\prime}}\right)^{(4)}$, i.e.,

$$
C_{f}=\frac{12}{R e_{b}}+12 \int_{0}^{1} 2(1-y)\left(\overline{u^{\prime} v^{\prime}}\right) d y,
$$

where the flow is fully developed and the quantities are made dimensionless by using twice the bulk-mean velocity and the channel half-width. In order to quantify the contribution of RSS, which depends on the distance from the wall, the weighted RSS (i.e., the integrand of the second term of Eq. (3)) is depicted in Fig. 3. The black solid line represents the 


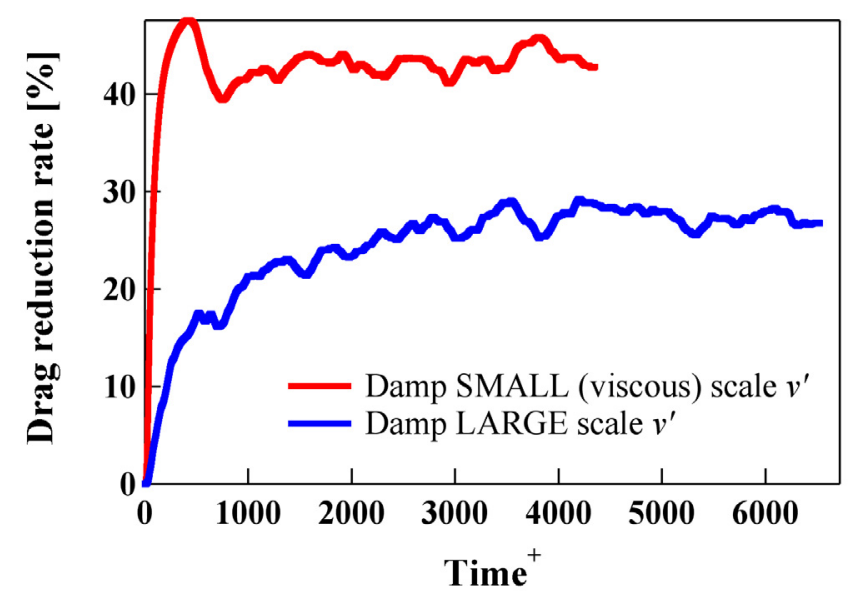

Fig. 2 Time trace of drag reduction rate

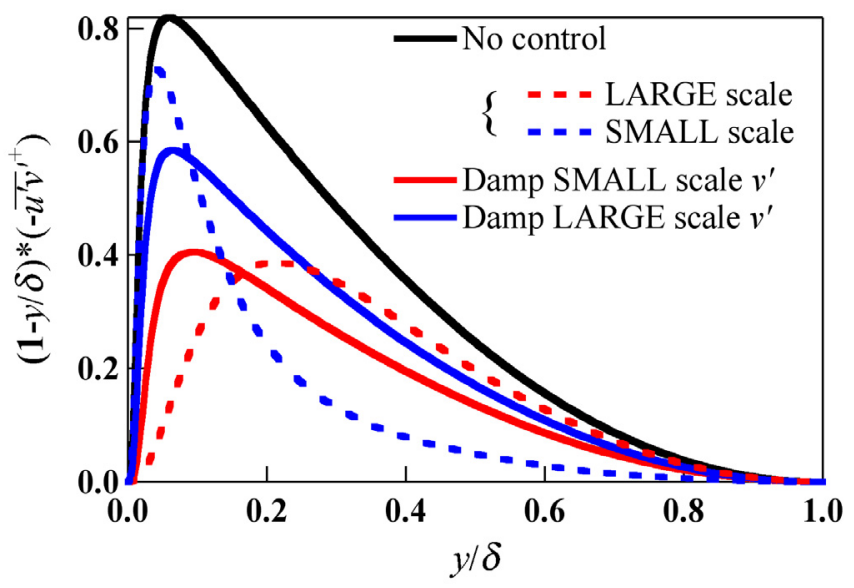

Fig. 3 Distribution of weighted Reynolds shear stress

weighted RSS (summation of all wavenumber components) in the uncontrolled flow. The red and blue dashed lines are the large-scale and small-scale components thereof, respectively. The red and blue solid lines represent the weighted RSS (summation of all wavenumber components) of the cases of small-scale and large-scale damping, respectively. As indicated by Eq. (3), the area enclosed by the curve of weighted RSS and the horizontal axis is the turbulent contribution term to the friction coefficient. Although both damping controls reduce the weighted RSS in entire channel, the effect of the small-scale damping (red solid line) is found to be larger.

One may intuitively expect that the turbulent contribution in the case of large-scale damping (i.e., the area enclosed by the blue solid line and the axis) is equal to that of the small-scale component in the uncontrolled flow (blue dashed line), and similarly, that in the case of small-scale damping (red solid line) is equal to that of the large-scale component in the uncontrolled flow (red dashed line). In the followings, we decompose the RSS in the controlled cases into the large-scale and small-scale components, and discuss their weighted integration (i.e., their contributions to friction drag).

The turbulent contribution term in Eq. (3) is decomposed into two scales: a small-scale contribution, i.e., the weighted integration of RSS due to the small-scale fluctuations $\left(\lambda_{z}^{+}<\right.$ $300)$, and the large-scale contribution, i.e., that due to the large-scale fluctuations $\left(\lambda_{z}^{+}>\right.$ 300). Figure 4(a) shows the contribution ratios from these two scales, which are defined as the contributions divided by the total friction drag of the uncontrolled flow. In the 
(a)

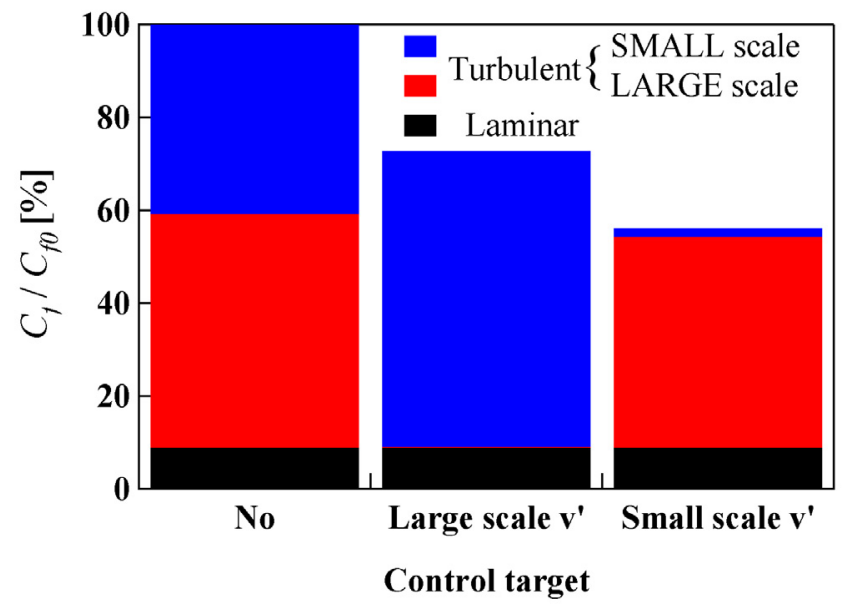

(b)

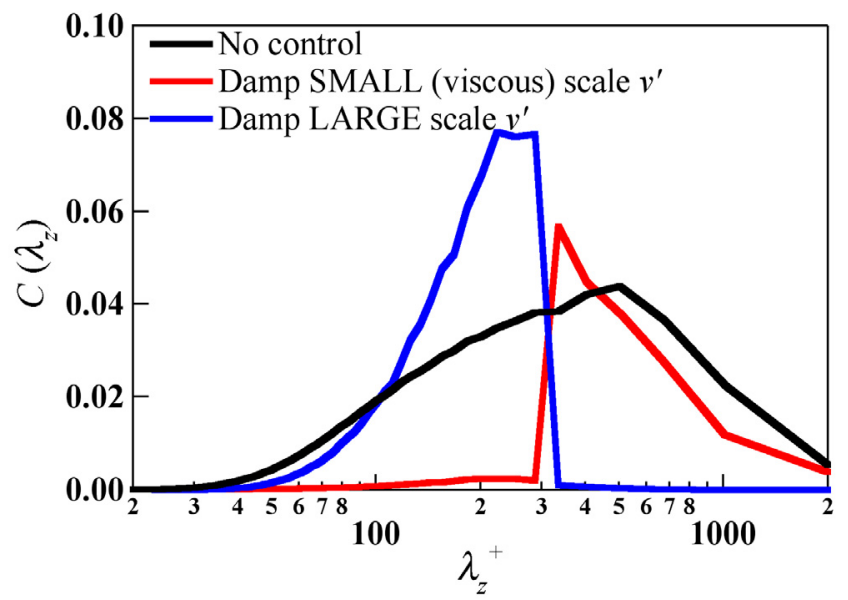

Fig. 4 (a) Contribution to friction drag; (b) Premultiplied co-spectra of turbulent friction term

uncontrolled flow, $91 \%$ of the friction drag is the turbulent contribution, of which about $55 \%$ is from the large-scale fluctuations sharing $2 / 3$ of the turbulent kinetic energy and $45 \%$ is from the small-scale fluctuations.

In the case of small-scale damping, the small-scale contribution reduces and the large-scale contribution remains unchanged. In the case of large-scale damping, in contrast, the large-scale contribution almost vanishes, but the small-scale contribution drastically increases. This explains why the larger drag reduction effect is achieved with the small-scale damping in the present numerical experiment.

Figure 4(b) shows the premultiplied co-spectrum of this turbulent contribution term, i.e.,

$$
C\left(\lambda_{z}\right) \equiv\left(\frac{\delta}{\lambda_{z}}\right) \int_{0}^{1}\left(1-\frac{y}{\delta}\right)\left(-\overline{\hat{u}^{\prime} * \hat{v}^{\prime}}\right) d\left(\frac{y}{\delta}\right),
$$

where the hat $(\hat{\therefore})$ denotes the spanwise Fourier transformation and the premultiplication factor $\left(\delta / \lambda_{z}\right)$ is applied so that the area displayed in the semi-log plot corresponds to the turbulent contribution term shown in Fig. 4(a). In the case of small-scale damping, the spectrum in the unmanupulated (i.e., large-scale) range is not significantly changed. In the case of large-scale damping, in contrast, the power in the small-scale range (especially its longer wave side) is found to significantly increase. In both cases, the power in the 
(a)

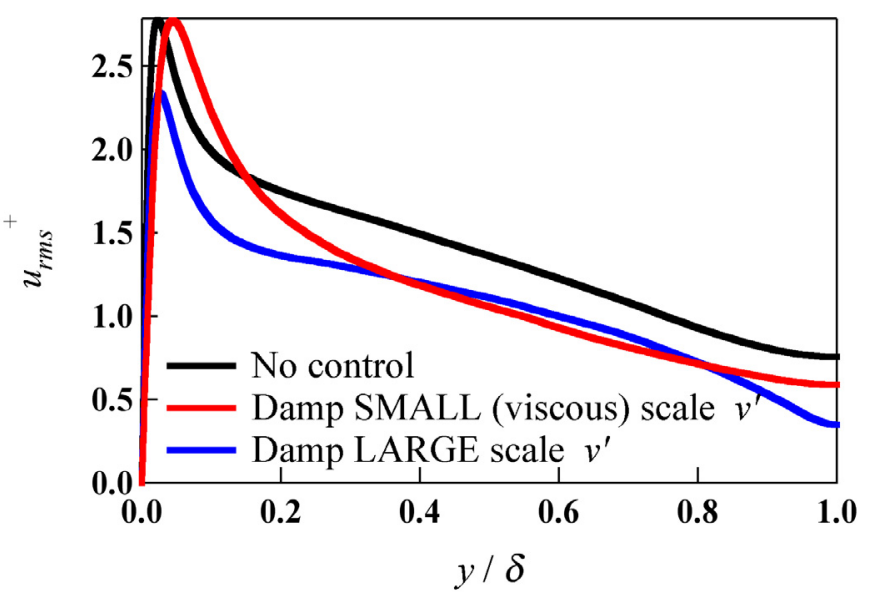

(b)

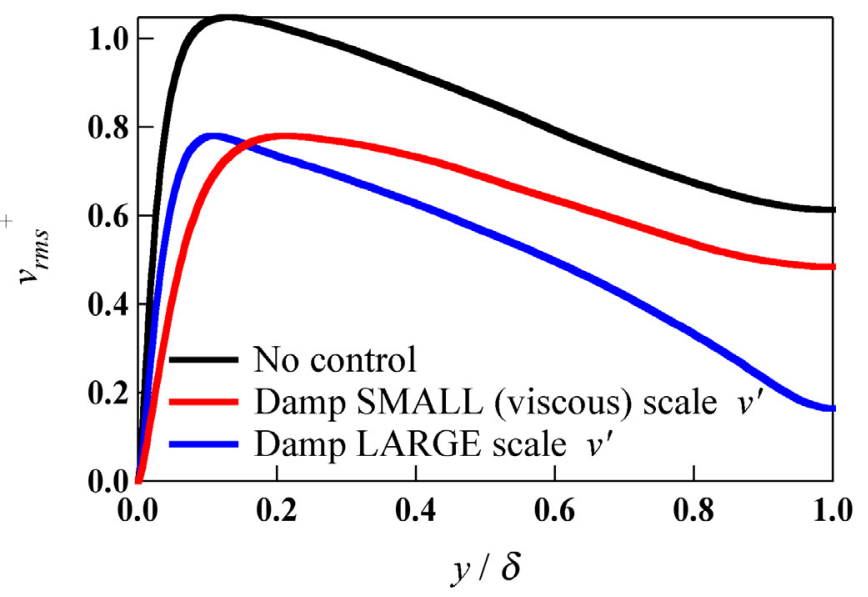

(c)

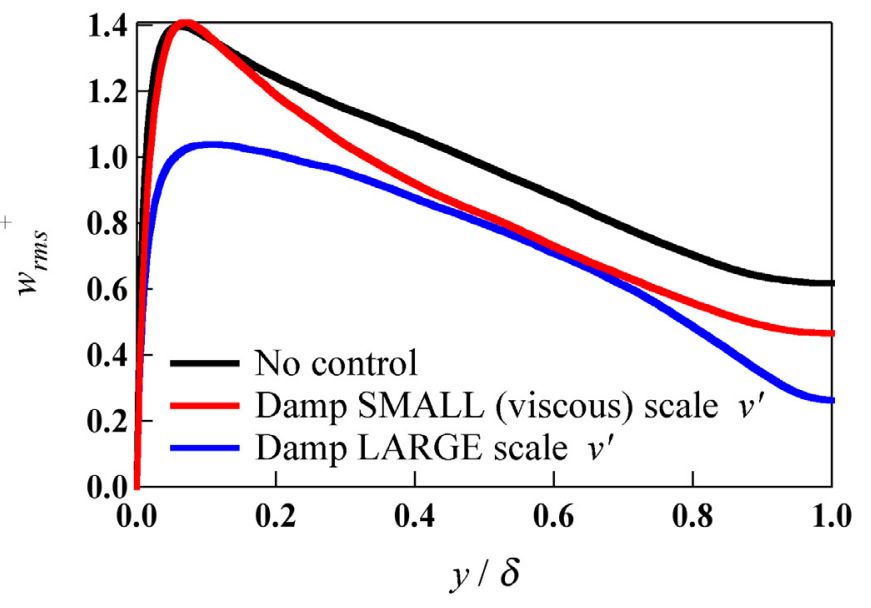

Fig. 5 Rms velocity fluctuations. (a) $u_{\text {rms }}$; (b) $v_{\text {rms }}$; (c) $w_{\text {rms }}$

manupulated wavenumber range is nearly zero, suggesting that the time constant of damping, $\tau=10 v / u_{\tau}^{2}$, used in the present simulation is sufficiently short to ideally damp the fluctuations of the specified wavenumbers.

Figure 5 shows the root-mean-square (RMS) values of velocity components in the cases of the large-scale and small-scale dampings. Unlike the change of RSS mentioned above, the RMS velocities are found to be smaller with the large-scale damping. This difference is 


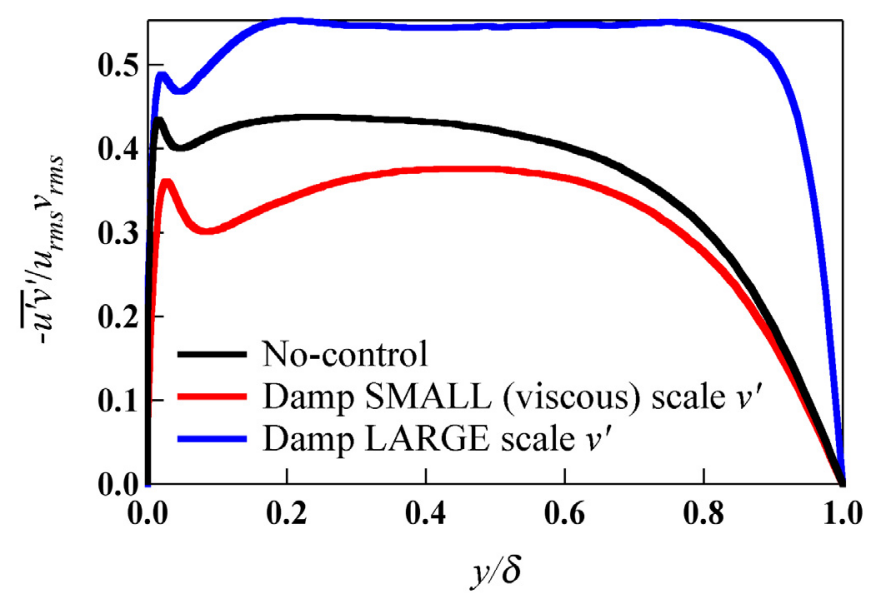

Fig. 6 Correlation coefficient of $-u$ 'v'

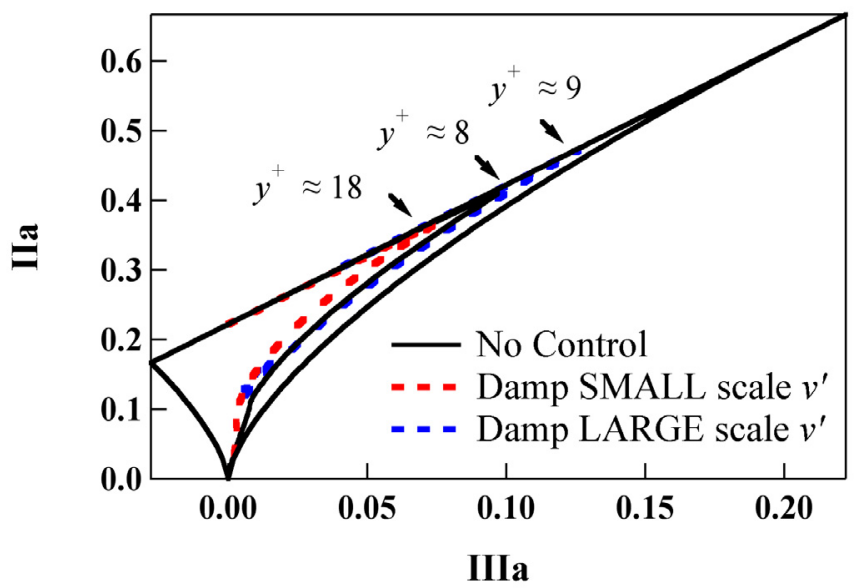

Fig. 7 Anisotropy invariant map

attributed to a stronger negative correlation between $u$ ' and $v^{\prime}$ as shown in Fig. 6.

In Fig. 7, the relationship between the second $\left(I I_{a}\right)$ and third invariants $\left(I I I_{a}\right)$ of the anisotropy tensor,

$$
\begin{aligned}
& a_{i j}=\frac{\overline{u_{i}{ }^{\prime} u_{j}{ }^{\prime}}}{\overline{u_{k}{ }^{\prime} u_{k}{ }^{\prime}}}-\frac{1}{3} \delta_{i j}, \\
& I I_{a}=a_{i j} a_{j i}, \\
& I I I_{a}=a_{i j} a_{j k} a_{j i},
\end{aligned}
$$

are depicted. Recently, Frohnapfel et al. ${ }^{(13)}$ have reported that the fluctuations approach the one-component limit (top right corner in the figure) in drag reducing flows by polymers or surfactants - typically, only the streamwise fluctuation is kept at the same level and the wall-normal and spanwise fluctuations as well as the Reynolds shear stress are suppressed. Such a common trend, however, is not observed in the present cases. As observed in the figure, the fluctuations approach the one-component limit in the case of large-scale damping, they depart from the limit in the case of small-scale damping.

This difference can be explained by the wall-normal location $y_{1 \mathrm{C}}$ where $I I_{a}$ takes the maximum value (see, Fig. 7) and the RMS value of each velocity component (Fig. 5), as 


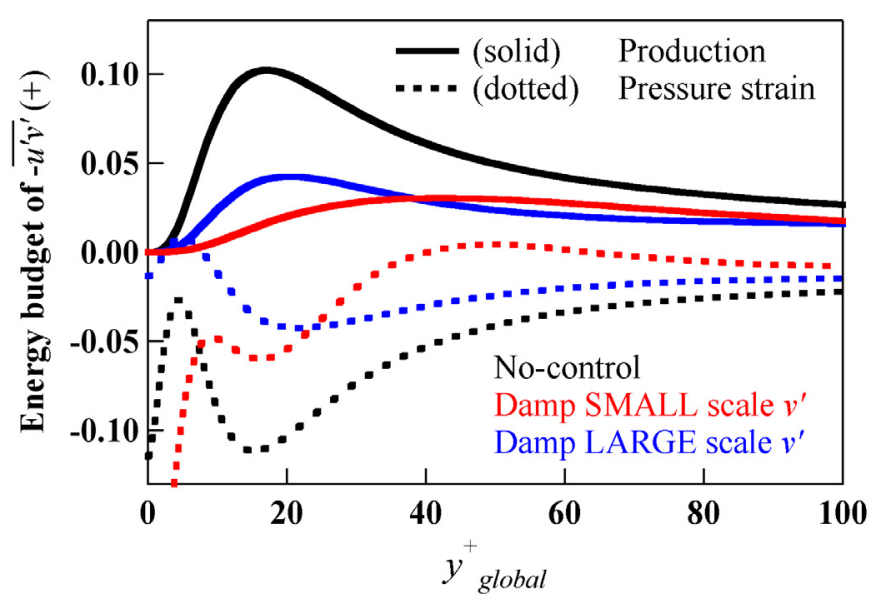

Fig. 8 Production and Pressure strain terms of $-\overline{u^{\prime} v^{\prime}}$ budget

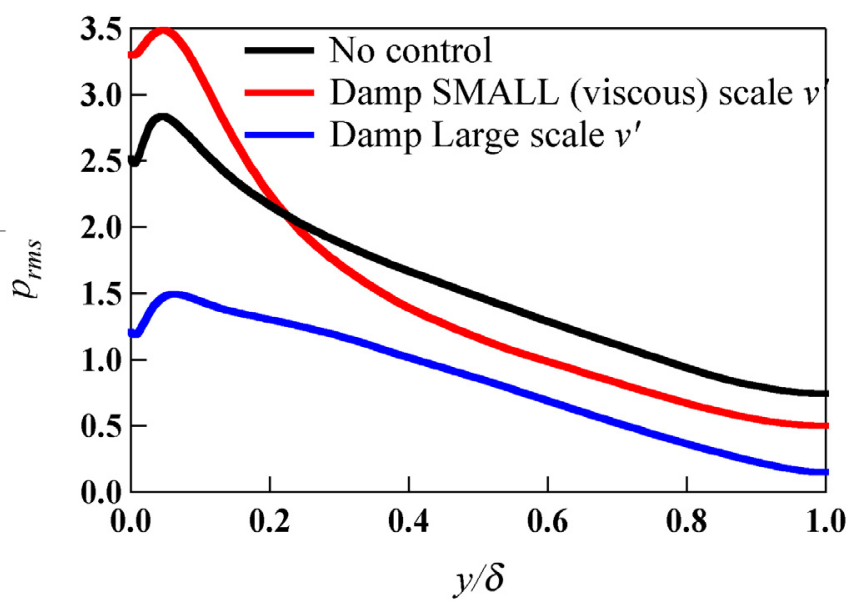

Fig. 9 Rms pressure fluctuations

follows. As compared to the uncontrolled flow, the peak location of $u_{\text {rms }}$, which is the largest component, moves away from the wall in the case of small-scale damping. Accordingly, $y_{1 \mathrm{C}}$ also moves away from the wall. As a result, the relative magnitude of $w_{\text {rms }}$ increases at the location of $y_{1 \mathrm{C}}$, leading to the departure from the one-component limit.

The reason for the increase of small-scale RSS components by the large-scale damping is examined by the budget of $\operatorname{RSS}\left(-\overline{u^{\prime} v^{\prime}}\right)$, which reads

$$
\begin{aligned}
\frac{D}{D t}\left(-\overline{u_{1}^{\prime} u_{2}^{\prime}}\right)= & \underbrace{\overline{u_{2}^{\prime 2}} \frac{\partial \overline{u_{1}}}{\partial x_{2}}-\frac{1}{\rho}\left(\overline{p^{\prime} \frac{\partial u_{1}^{\prime}}{\partial x_{2}}+\overline{p^{\prime}} \frac{\partial u_{2}^{\prime}}{\partial x_{1}}}\right)}_{\langle\mathrm{P}\rangle}+\underbrace{\frac{1}{\rho}\left\{\frac{\partial\left(\overline{u_{1}^{\prime} p^{\prime}}\right)}{\partial x_{2}}+\frac{\partial\left(\overline{u_{2}^{\prime} p^{\prime}}\right)}{\partial x_{1}}\right\}}_{\langle\mathrm{PS}\rangle} \\
& +\underbrace{v \frac{\partial^{2}\left(-\overline{u_{1}^{\prime} u_{2}^{\prime}}\right)}{\partial x_{k} \partial x_{k}}+\underbrace{\frac{\partial\left(\overline{u_{1}^{\prime} u_{2}^{\prime} u_{k}^{\prime}}\right)}{\partial x_{k}}}_{\langle\mathrm{TD}\rangle}-\underbrace{2 v \frac{\partial u_{1}^{\prime}}{\partial x_{k}} \frac{\partial u_{2}^{\prime}}{\partial x_{k}}}_{\langle\mathrm{D}\rangle} .}_{\langle\mathrm{VD}\rangle}
\end{aligned}
$$

Figure 8 shows the distributions of production (P) and pressure-strain correlation (PS) terms. It is found that in the case large-scale damping the near-wall production is stronger and the pressure-strain correlation is weaker than those in the case of small-scale damping. The weaker pressure-strain correlation with the large-scale damping is attributed to the smaller 
(a)

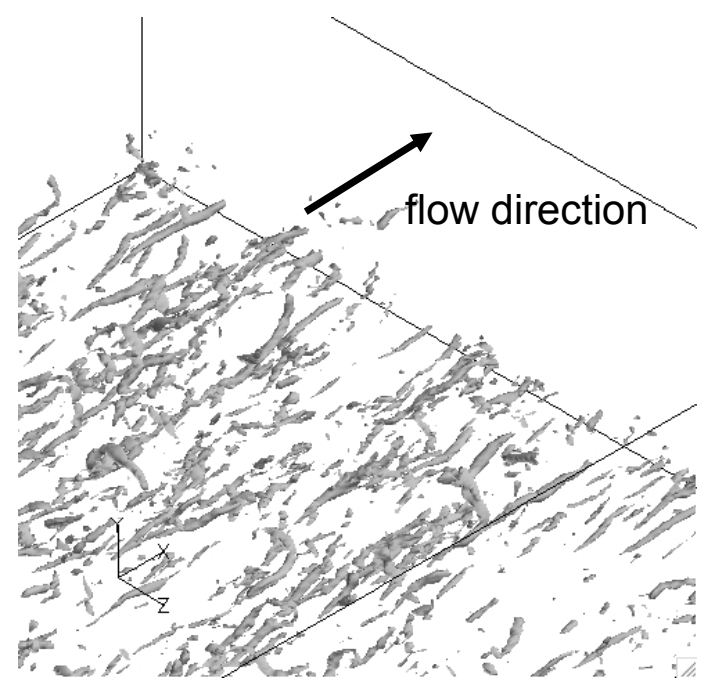

(b)

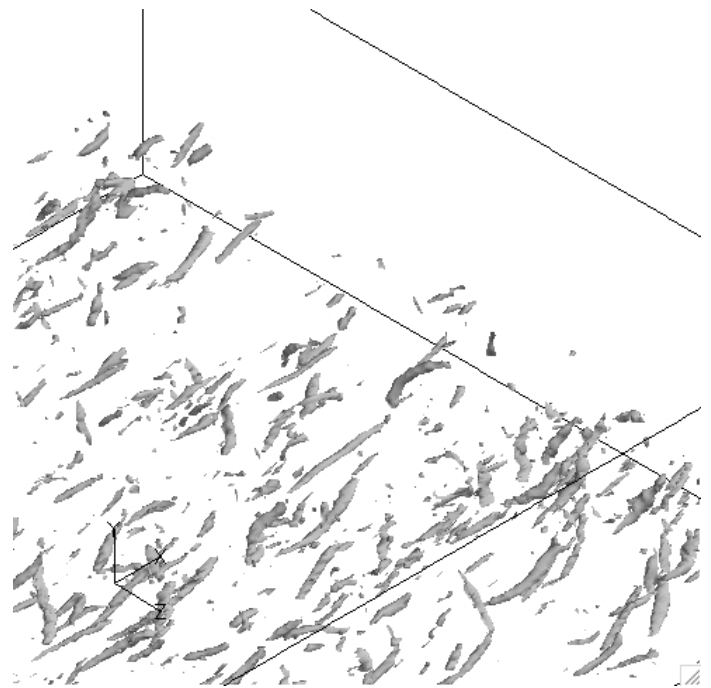

(c)

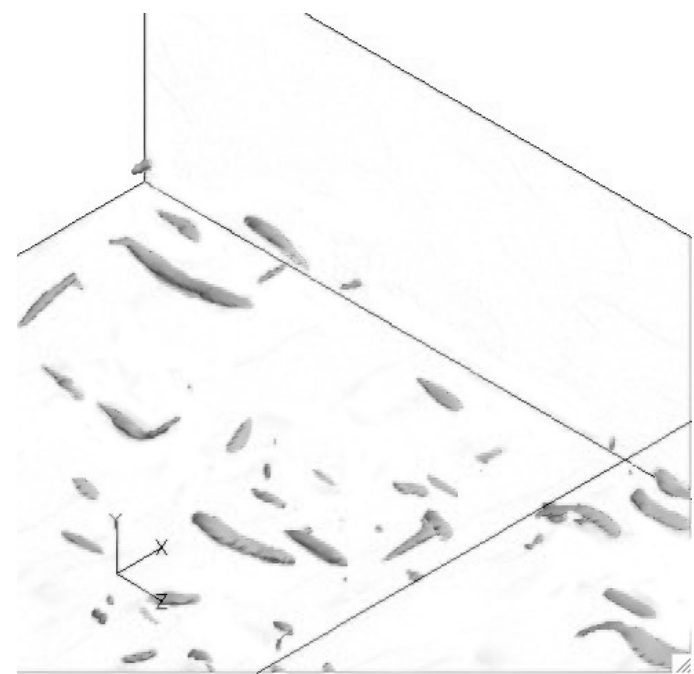

Fig. 10 Instantaneous flow field. (a) No control $\left(I I^{+}=-0.05\right)$; (b) Damp large scale $v^{\text {' }}$ $\left(I I^{+}=-0.03\right)$; (c) Damp small scale $v^{\prime}\left(I I^{+}=-0.015\right)$. Bottom plane: wall, Upper plane: channel center. 
pressure fluctuations as shown in Fig. 9. The smaller pressure fluctuation by the large-scale damping is natural considering the fact that pressure fluctuations have more power in the lower wavenumber range (i.e., larger structures) in the uncontrolled flow (figure omitted). As clearly illustrated in Kasagi et al., ${ }^{(14)}$ the pressure fluctuation plays an important role to destruct the RSS. Therefore, the increase of small-scale contribution by the large-scale damping can be attributed to that the destruction of RSS is suppressed by the suppression of pressure fluctuation.

Figure 10 illustrates the instantaneous vortical structures in each case. The vortical structures are identified by using the second invariant of the deformation rate tensor, $I I$. A number of quasi-streamwise vortices are identified in the case of large-scale damping. In the case of small-scale damping, in contrast, quasi-streamwise vortices are scarcely observed, but spanwise vortices are identified instead. From the streamwise two-point correlation of wall-normal velocity (figure omitted), these spanwise vortices are found to have the spacing of $80-200 v / u_{\tau}$.

\section{Conclusions}

Various feedback control methods have been proposed aiming at friction drag reduction of wall-turbulence. For their practical use, however, one has to overcome the technical hurdles in fabrication, operation, and economic use of the micro-sized sensors/actuators required. In the present study, we examined the possibility of using larger actuators than those assumed in the conventional control laws. Namely, we performed numerical experiments of fully developed turbulent channel flow with selective damping of either large-scale or small-scale fluctuations.

We applied a feedback body force in entire channel so as to damp the wall-normal velocity fluctuations. The damping of small-scale fluctuations (small-scale damping) is found to reduce the friction drag more effectively than the damping of large-scale fluctuation (large-scale damping). While the contribution of large-scale fluctuations to the friction drag is nearly unchanged in the case of small-scale damping, the contribution of small-scale fluctuations significantly increases in the case of large-scale damping. The increase of small-scale fluctuations in latter case is attributed to the suppression of destruction of Reynolds shear stress due to weakened pressure fluctuations.

Since the large-scale structures have larger contribution to the friction drag in high Reynolds number turbulent flows, we initially expected that the manipulation of large-scale structures might be as effective as that of small-scale structures. However, the present simulation results suggest that the possibility we can attain a larger drag reduction effect by the manipulation of large-scale structures than that by the conventional control strategy (i.e., the manipulation of near-wall small-scale structures) is quite low.

\section{Acknowlegments}

The authors are grateful to Dr. Kaoru Iwamoto (Tokyo University of Agriculture and Technology) for his advice on numerical procedure and Dr. Yuji Suzuki (The University of Tokyo) for fruitful discussion. This work was supported partly through Grant-in-Aid for Scientific Research (A) by Japan Society for the Promotion of Science (JSPS).

\section{References}

(1) Choi, H., Moin, P. and Kim, J., Active turbulence control for drag reduction in wall-bounded flows. J. Fluid Mech., Vol. 262 (1994), pp. 75-110. 
(2) Morimoto, K., Iwamoto, K., Suzuki, Y. and Kasagi, N., Genetic algorithm-based optimization of feedback control scheme for wall turbulence. Proc. 3rd Symp. Smart Control of Turbulence (2002), pp. 107-113.

(3) Fukagata, K. and Kasagi, N., Suboptimal control for drag reduction via suppression of near-wall Reynolds shear stress. Int. J. Heat Fluid Flow, Vol. 25 (2004), pp. 341-350.

(4) Yoshino, T., Suzuki, Y. and Kasagi, N., Feedback control of turbulence air channel flow with distributed micro sensors and actuators. J. Fluid Sci. Technol., Vol. 3 (2008), pp. 137-148.

(5) Tomkins, C. D. and Adrian, R. J., Energetic spanwise modes in the logarithmic layer of a turbulent boundary layer. J. Fluid Mech., Vol. 545 (2005), pp. 141-162.

(6) Kasagi, N., Suzuki, Y. and Fukagata, K., Microelectromechanical system-based feedback control of turbulence for skin friction reduction. Annu. Rev. Fluid Mech., Vol. 41 (2009), pp. 231-251.

(7) Abe, H., Kawamura, H. and Choi, H., Very large-scale structures and their effects on the wall shear-stress fluctuations in a turbulent channel flow up to $\mathrm{Re}_{\tau}=640$. Trans. ASME J. Fluids Eng., Vol. 126 (2004), pp. 835-843.

(8) Iwamoto, K., Kasagi, N. and Suzuki, Y., Dynamical roles of large-scale structures in turbulent channel flow. Computational Mechanics, WCCM VI in conjunction with APCOM'04, Sept. 5-10, 2004, Beijing, China, MS022-174 (2004).

(9) Iwamoto, K., Fukagata, K., Kasagi, N. and Suzuki, Y., Friction drag reduction achievable by near-wall turbulence manipulation at high Reynolds number. Phys. Fluids, Vol. 17 (2005), Art. 011702.

(10) Kim, J., Moin, P. and Moser, R. D., Turbulence statistics in fully developed channel flow at low Reynolds number. J. Fluid Mech., Vol. 177 (1987), pp. 133-166.

(11) Smith, C. R. and Metzler, S. P., The characteristics of low-speed streaks in the near-wall region of a turbulent boundary layer. J. Fluid Mech., Vol. 129 (1983), pp. 27-54.

(12) Fukagata, K., Iwamoto, K. and Kasagi, N., Contribution of Reynolds stress distribution to the skin friction in wall-bounded flows. Phys. Fluids, Vol. 14 (2002), pp. L73-L76.

(13) Frohnapfel, B., Lammers, P., Jovanović, J., and Durst, F., Interpretation of the mechanism associated with turbulent drag reduction in terms of anisotropy invariants. J. Fluid Mech., Vol. 577 (2007), pp. 457-466.

(14) Kasagi, N., Suzuki, Y. and Iida, O., Kinematics of the quasi-coherent vortical structure in near-wall turbulence. Int. J. Heat Fluid Flow, Vol. 16 (1995), pp. 2-10. 